\title{
Combination of histological and molecular data for improving outcome prediction in non-muscle invasive bladder cancer- narrative review
}

\author{
Radosław Piszczek ${ }^{1} \wedge$, Wojciech Krajewski ${ }^{2}$, Marco Moschini ${ }^{3}$, Anna Kołodziej ${ }^{2}$, Łukasz Nowak $^{2}$, \\ Adrian Poterek ${ }^{2}$, Romuald Zdrojowy ${ }^{2}$
}

${ }^{1}$ Department of Urology and Oncological Urology, Lower-Silesian Specialistic Hospital, A.E. Fieldorfa 2, Wrocław, Poland; ${ }^{2}$ Department of Urology and Oncological Urology, Wrocław Medical University, Borowska 213, Wrocław, Poland; ${ }^{3}$ Department of Urology, Luzerner Kantonsspital, Spitalstrasse 6004 Luzern, Switzerland

Contributions: (I) Conception and design: R Piszczek, W Krajewski; (II) Administrative support: None; (III) Provision of study materials or patients: None; (IV) Collection and assembly of data: None; (V) Data analysis and interpretation: None; (VI) Manuscript writing: All authors; (VII) Final approval of manuscript: All authors.

Correspondence to: Radosław Piszczek. Department of Urology and Oncological Urology, Lower-Silesian Specialistic Hospital, A.E. Fieldorfa 2, Wrocław, Poland. Email: piszczek.radoslaw@gmail.com.

\begin{abstract}
The majority of patients with bladder cancer are diagnosed in non-muscle invasive stage. Most of them will experience recurrence or progression to more aggressive disease during follow-up. That raises the need for improvements with regard to risk assessment. Current risk stratification, based only on clinicopathologic features, does not fully reflect biological heterogeneity of the cancer and its role in prognosis. Many studies addressed the topic of variant histology and its influence on treatment and outcomes. It has been shown that accurate identification of variant histology implicates patient prognosis and inform right treatment decisions. Most studies on histological variants of bladder cancer suggest a more aggressive clinical course, with higher risk of recurrence and progression than in conventional urothelial cancer, even when diagnosed in non-muscle invasive stage. That prompts early aggressive treatment approach whenever variant histology is detected. Emerging genomic information are expected to complement clinical and pathological data and change the paradigms in the management of bladder cancer. Several reports highlighted the clinical significance of molecular stratification of bladder cancer, but the available evidence is based on retrospective data. Molecular subtyping gives promise not only for improving risk assessment, but also in predicting response to Bacillus Calmette-Guerin (BCG) or chemotherapy. Finally, molecular alterations might become targets for novel drugs to improve the overall response of these patients. However, its implementation into clinical practice requires further validation in prospective trials, especially in the context of non-muscle invasive bladder cancer.
\end{abstract}

Keywords: Non-muscle invasive bladder cancer; variant histology; molecular variants

Submitted Jun 04, 2020. Accepted for publication Oct 09, 2020.

doi: $10.21037 /$ tcr-20-2257

View this article at: http://dx.doi.org/10.21037/tcr-20-2257

$\wedge$ ORCID: 0000-0002-7505-6338. 


\section{Introduction}

According to epidemiological data, bladder cancer (BC) is 7 th most prevalent cancer in males and 11 th in both genders (1). Approximately $75 \%$ of patients are diagnosed with non-muscle invasive bladder cancer (NIMBC), while the remaining present with muscle-invasive disease. Almost $70 \%$ of patients with NIMBC will develop recurrence after treatment of primary tumor, and up to $30 \%$ will progress into more aggressive disease (2). Several factors have been linked with the risk of $\mathrm{BC}$ development. Active and passive smoking are the most relevant risk factors, found almost in half of the cases (3). Occupational exposure to aromatic amines, polycyclic aromatic hydrocarbons and chlorinated hydrocarbons is the second most important risk factor for BC, accounting for up to $25 \%$ of all cases (4). Bladder schistosomiasis and other chronic urinary tract infections have been shown to increase the risk of BC. There is also a well-described relationship between Bilharzia and squamous cell variant of $\mathrm{BC}(3,5)$. Increased rates of secondary bladder carcinomas have been reported after radiotherapy for pelvic malignancies (3). There is also difference in BC prevalence between both genders, with higher incidence among men, however women present with more advanced disease and have worse survival rates (3). There is growing evidence that genetic factors and family association may influence the incidence of BC. Several susceptibility loci have associated with $\mathrm{BC}$ risk have been identified (6). Genetic predisposition may also influence the incidence of BC through its impact on susceptibility to other risk factors $(3,6)$. Possible association was also suggested for the use of cyclophosphamide and pioglitazone. The role of dietary as well as metabolic factors remains uncertain (3).

The white-light cystoscopy of the bladder with histological evaluation of the tissue obtained by either cold-cup biopsy or transurethral resection (TURB) are the current standard in diagnosis and primary treatment of BC (7). Given the high overall risk of tumor recurrence, strict follow-up is recommended with regular cystoscopy and resection in case of relapse. Several intravesical treatment modalities have been proposed in order to reduce recurrence and prevent progression do muscleinvasive stage, with Bacillus Calmette-Guerin (BCG) instillations being the most efficient one (8). Treatment and surveillance strategies for patients with bladder cancer are currently dictated by histologic evaluation of resected tissue, including determination of tumor grade and stage. Current risk stratification in NMIBC is based on European
Organisation for Research and Treatment of Cancer (EORTC) and Spanish Urological Club for Oncological Treatment (CUETO) scoring systems $(9,10)$. According to these nomograms, three prognostic subgroups of low, intermediate and high-risk can be distinguished which directly influences treatment decisions $(9,10)$. In this setting, prognosis relies on clinicopathologic features that do not reflect the biological heterogeneity of the cancer.

It is becoming more apparent that reliable evaluation of the tumor's aggressiveness and optimal management of BC requires accurate assessment of the tumor's biological characteristics. Many studies addressed the topic of variant histology $(\mathrm{VH})$ in the context of muscle-invasive (MIBC) and metastatic BC, and its influence on treatment and outcomes. Despite the large prevalence of NMIBC, data concerning the effect of variant histology on prognosis is scarce and true clinical variant histology significance is controversial.

Along VH, molecular subtyping is another emerging diagnostic modality with the potential for improving prediction of recurrence and progression in NMIBC. Over the years, several biomarkers have been identified for prognostic purposes, such as mRNA gene expression signatures, panels of epigenetic and immunohistochemistry markers in order to complement the pathologic evaluation and improve prognosis in NMIBC. Based on molecular data, several retrospective studies proposed tumor molecular sub classification and associated these subtypes with different prognoses.

It seems promising that the combination of histological and molecular information can be more accurate for predicting clinical outcome. Most of available literature focuses on the topic mainly in the context of MIBC or both MIBC and NIMBC, whilst studies regarding NMIBC alone are lacking. In this review, we present the most relevant findings regarding both histological and molecular variants, specifically in the setting of NMIBC, that have been reported over the recent years. We present the following article in accordance with the Narrative Review reporting checklist (available at http://dx.doi.org/10.21037/tcr-20-2257).

\section{Relevance of TURB and pathology in diagnosis of histological variants and molecular evaluation}

Diagnosis of histological variants, as well as correct molecular subtyping, relies strongly on the amount and quality of tissue obtained during TURB. Several factors can influence the quality of resected samples. Firstly, 
NMIBCs can be very small in size which makes it difficult to obtain enough representative tissue for histological and thorough genomic analysis. Secondly, the use of cautery and resulting burning effect can cause tissue degradation, making the reliable evaluation challenging. The operation time has been also adversely associated with the extent of mRNA degradation (11). Another important factor is the method of tissue preservation. It has been shown that in frozen samples mRNA is relatively stable, while when formalin-fixed and paraffin-embedded, mRNA is often severely degraded (11). Also, it has to remembered that specimens can contain a large number of other components, such as immune or stromal cells. Therefore, an improper purification of resected tissue can affect the results of molecular subtyping (12). Finally, quality of histological or molecular subtyping can be affected by intratumoral heterogeneity. A study by Warrick et al. clearly showed that tissue samples obtained from different tumour sites can present different molecular patterns (13). Similarly, a coexistence of different histological variants within the same tumour has been described (14). The importance of resection quality with regard to $\mathrm{VH}$ has been addressed in several studies. In a report by Cai et al., authors evaluated TURB specimens of patients undergoing radical cystectomy (RC) and found significant disagreement between TURB and radical cystectomy specimens. Out of 4,110 patients, 579 were found to have $\mathrm{VH}$ in cystectomy specimens, while after TURB variant histology was confirmed in 266 patients (15). Similarly, Moschini et al. analyzed a group of 779 patients treated with TURB with subsequent cystectomy. A low concordance between the two specimens was found in general and depended on the type of variant (16). In contrast to these reports, Abufaraj et al. reviewed 268 patients who underwent TURB with subsequent radical cystectomy, and found $83.6 \%$ agreement between the histopathological reports (17).

Having considered all these elements, obtaining highquality samples is the crucial step to reliably classify tumours on the basis of their histological and molecular characteristics.

\section{Prognostic role of histological variants in NIMBC}

The heterogeneity and wide variety of histopathological patterns have been well described for urothelial carcinoma. The term variant histology includes urothelial cancer (UC) with presence of other morphologies, such as urothelial cancer with mixed histologic features as well as pure non- urothelial cancers (1). In this review, we analyzed data regarding coexistence of divergent morphology with conventional urothelial component. The 2016 WHO Classification of Tumours of the Urinary System and Male Genital Organs emphasized prognostic importance of variant histology by distinguishing the category of $\mathrm{UC}$ with divergent differentiation. According to this classification, bladder cancers can be classified as urothelial and nonurothelial tumors (2). There is a consensus that the presence of divergent morphology and some estimate of its quantity should be reported.

Squamous differentiation is the most common histological variant with estimated incidence up to $40 \%$ of invasive BCs and mostly associated with Bilharzia infection with higher frequency in Middle East countries (18). However, non-bilharzial squamous pattern can occur in Western populations and has been linked to recurrent urinary tract infections, bladder stones, indwelling catheters, pelvic radiation therapy, intravesical treatment with BCG, as well as exposure to cyclophosphamide (19-21). Histologically, it is defined by the presence of intercellular bridges or keratinization $(14,22)$. Squamous differentiation has to be distinguished from pure squamous cell carcinoma in which no urothelial carcinoma component can be found (23). True incidence of squamous differentiation in NMIBC is unknown, owing to the fact that this pattern in low - stage tumours is often focal and can be often missed during conventional evaluation (23).

Squamous patterns detected in the histopathological specimen represents a negative prognostic factor. In a study by Lopez-Beltran et al. (23), it was found that $\mathrm{Ta} /$ T1 tumours with squamous differentiation carry a greater risk of recurrence than pure UC. However, no association was found in terms of survival, suggesting that its effect on survival status is probably related to higher grade and stage (23).

In a paper by $\mathrm{Li}$ et al., a retrospective analysis was conducted in order to assess the efficacy of intravesical chemotherapy in T1 tumours with or without squamous component. Treatment protocol comprised of initial TURB and subsequent intravesical chemotherapy with epirubicin or hydroxycamptothecine. In this study, squamous differentiation of $\mathrm{pT} 1$ bladder UC was associated with higher tumour recurrence and progression rates, especially for high-grade tumours. It suggests that this variant might predict poor response to intravesical chemotherapy. Yet, no clear difference regarding survival was found (24).

In another study evaluating the efficacy of intravesical 
therapy in variant histology NIMBC, it was found that patients with variant bladder cancer, including squamous pattern, had significantly worse prognosis in terms of progression, recurrence and survival, compared to patients with pure urothelial tumors (25).

These findings are somehow consistent with the results of several studies on MIBC in which squamous differentiation was found to be associated with higher progression rates and worse oncological outcomes (26,27). A poor response to chemotherapy or radiation therapy was shown in some studies, which once more may be associated with advanced stage of the disease rather than histology itself.

In contrast to abovementioned studies, some authors reported comparable survival rates between patients with squamous differentiation and those with pure UC, when adjusted for tumour stage $(28,29)$. Interestingly, the importance of early diagnosis and aggressive surgical treatment was underlined by the SEER database (30). In this analysis, a subgroup of patients with squamous cell carcinoma was reviewed, and it was found that among those with T1 disease who did not undergo radical cystectomy, a squamous subtype was a strong and independent predictor of poor cancer specific survival. Athwart, among cases with stage $\mathrm{T} 1$ in which the bladder was removed as a part of the initial treatment, squamous histologic features were not associated with increased mortality when compared to pure $\mathrm{UC}(30)$.

\section{Treatment of SCC}

The precise data regarding management of squamous differentiation in non-muscle invasive disease are lacking. Bearing in mind that the presence of squamous component might carry a higher risk of recurrence and progression, as well as poorer response to intravesical chemotherapy, early radical cystectomy (RC) is considered the most suitable treatment option (24-26).

In the previously mentioned study by Gang $\mathrm{Li}$, it was concluded that patients with high-grade and recurrent NMIBC with squamous differentiation should undergo early RC to prevent disease progression (24).

The efficacy of intravesical therapy in variant histology NIMBC has been evaluated by Gofrit et al. A group of 41 patients received BCG immunotherapy. The results suggest inferior performance in tumors with squamous differentiation with progression rates to muscle-invasive disease of up to $45 \%$ within 2 years and subsequent death in almost all patients with progression (25).

Urothelial cancers with glandular differentiation are the second most common form of divergent differentiation, seen in up to $18 \%$ of all BCs (31). Pathologically, they are defined by the presence of gland formation within the tumour which may have tubular, enteric, signet-ring and villous-like appearance. UCs with glandular differentiation may be seen occasionally associated with urothelial CIS with glandular differentiation. Similarly to squamous variant, a tumour with mixed glandular and urothelial component is classified as UC with glandular differentiation. Therefore, patients with only glandular differentiation should be labelled as adenocarcinoma $(32,33)$.

The meaning of glandular differentiation in NIMBC in terms of poorer outcomes is debatable, as there is paucity of literature on this variant with small groups of patients being reported. Most published reports have not assessed glandular differentiation in isolation, but rather in a combination with squamous pattern.

The largest series of NMIBC with glandular differentiation was reported by Miller and Epstein (34). In that series, 6 out of 7 patients treated with BCG were eligible for follow-up. None of them developed recurrence. Similar results were achieved by Shapur et al., who reported group of only 4 patients with glandular variant non-invasive tumours. After a median follow-up of 22 months, none developed recurrence or progression (35).

In a series by Mitra et al., after matching for stage and percentage of variant, similar survival outcomes were reported in patients with glandular differentiation compared to patients with pure UC (36). In a study by Kim et al., similar survival outcomes between tumours with glandular differentiation and conventional UC were found after stage adjustment, however, patients with squamous and/or glandular differentiation were more likely to have extravesical tumours and node positive disease (37).

Although data regarding treatment are sparse, BCG instillations seem to outperform other forms of intravesical therapies and might be performed in patients with glandular variant of NMIBC (38). It has to be emphasized, that this is not the case in pure adenocarcinoma which is considered an aggressive disease and should be treated aggressively with RC.

Sarcomatoid variant is another rare variant with reported incidence that ranged from $0.3 \%$ of all urothelial cancers (39). However, some authors report that sarcomatoid foci can be found in up to $6 \%$ of UC (40). This type exhibits features of both epithelial and mesenchymal 
differentiation. Due to highly variable morphology, the diagnosis is often challenging (41). This variant frequently presents at an advanced stage and has worse overall survival when compared with pure UC, even after adjustment for stage $(42,43)$. There are no reports that assessed outcomes of TURB alone or with intravesical instillation. One study concluded that RC should be the preferred option for patients with T1 disease, rather than intravesical therapy (44). Other reports confirm the importance of early radical treatment, preferably radical cystectomy without neoadjuvant chemotherapy as it did not show any clinical benefit (45).

Micropapillary variant has a prevalence of about $0.6-1 \%$ of all BCs with significant male predominance. Microscopically, it is characterized by small nests and aggregates of tumour cells within lacunae without vascular cores (2). This variant often presents at high pathologic stage and has low survival rates. However, studies comparing outcomes after RC did not find increased recurrence rates or adverse cancer-specific survival in micropapillary carcinoma, when compared to pure UC (46). Relatively high proportion of micropapillary component has been shown in NMIBC with most patients presenting with $\mathrm{T} 1$ or CIS lesions $(47,48)$. The role of bladder sparing treatment in this histological variant has been examined. In a study by Kamat et al. (47), a high incidence of BCG failure was reported with $67 \%$ progression rate occurring at median of 8 months. Among patients treated with RC 5and 10 -year overall survival was $71 \%$ and $53 \%$, respectively. None of the patients treated with bladder preservation strategy survived 10 years (47). Similar observations were achieved by Spaliviero et al. who analyzed a group of 36 cases with either T1 or CIS with micropapillary component. Patients were managed by either early RC or BCG instillations with deferred cystectomy. The reported 5 -year cancer specific mortality was $25 \%$ in the group managed conservatively, compared to $17 \%$ in those who were treated with early RC (49). Willis et al. (48) reported a group of 72 patients treated with either BCG or up-front cystectomy. A 5-year disease specific survival was $100 \%$ for patients undergoing cystectomy, whereas $60 \%$ in the group managed conservatively. In addition, almost half of the patients treated with bladder preserving protocol developed progression (48). In contrast to these results, Jackson et al. retrospectively reviewed the outcomes of 40 non-invasive tumors with micropapillary features in order to identify patients who might be managed conservatively (50). The cohort was divided into two groups of superficial and invasive micropapillary differentiation. It was reported that superficial type is associated with higher median overall of 63 months, compared to 47 months in the second group. Thus, authors concluded that detailed pathological analysis may help identify patients with non-invasive micropapillary tumors that could be managed without immediate RC (50). A meta-analysis by Abufaraj et al. showed significantly lower survival rates for patients with T1 micropapillary bladder cancer treated with TURB plus BCG instillation, compared to those managed by early-cystectomy (51). In a recently published EAU-ESMO consensus, most experts agreed that $\mathrm{RC}$ with lymph node dissection represents the optimal approach in the treatment of T1 high-grade bladder UC with micropapillary histology (52).

Plasmocytoid variant is a rare and aggressive variant which is characterized by the presence of mononuclear tumour cells with plasmacytoid, lymphoid, or even rhabdoid features. Also, single cells with cytoplasmic vacuoles that impart a signet ring cell appearance are commonly seen in this variant (53). At the time of diagnosis, it usually occurs as extravesical disease with distant metastasis and is associated with poor prognosis (54). In a recent study by $\mathrm{Li}$ et al., a group of 98 patients with plasmocytoid carcinoma undergoing cystectomy was analysed. Plasmocytoid features were associated with advanced stage and higher rate of positive lymph nodes and positive margins. However, this was not associated with worse survival outcomes when compared with patients with pure UC (55). Therefore, RC with chemotherapy should be considered as a treatment of choice (56).

A nested variant represents another rare entity with relatively small numbers reported in the literature. Histologically, it is characterized by bland tumour cells that can form solid expansile or infiltrative nests or tubules (57). The true incidence of nested pattern in NMIBC is unknown, and so is the optimal management. It usually presents at advanced stage with muscular and nodal invasion and only up to $30 \%$ of nested variant cases are non-muscleinvasive at the time of diagnosis. It may be related to the late diagnosis frequently associated with this tumour $(58,59)$. This variant can imitate benign urothelial proliferations, especially in superficial transurethral resections and coldcup biopsies, thus some authors underlined the importance of restaging TURB to confirm non-muscle invasive status (59). Mally et al. retrospectively reviewed 30 patients with stage T1 tumours with nested features in order to assess if there is a difference in progression, metastasis or cancerspecific survival compared to patients with pure UC (59). 
No major differences in these parameters were found after matching for stage. However, patients with T1 disease in restaging TURB had higher rates of upstaging and nodal metastases in cystectomy specimens, compared to those with Ta disease. Still, after surgical treatment with an early cystectomy, there was no survival difference between nested variant tumors and pure UC. Authors also concluded that patients with $<\mathrm{T} 1$ in restaging TURB may be considered for conservative management (59). In study by Gofrit et al. evaluating the response to intravesical immunotherapy in $\mathrm{VH}$ tumors, a subgroup of 7 nested variant non-invasive tumors was reported. It was shown that this pattern had significantly worse prognosis compared to patients with conventional tumors in recurrence, progression and survival rates (25).

Next VH of UC is a trophoblastic differentiation with elevated beta-human chorionic gonadotropin levels. These tumours are rare and have been associated with a poor prognosis. Elevated levels of beta-HCG have been linked to poor response to chemo- and radiotherapy $(60,61)$. Up to date, there has been no study comparing tumours of similar grade and stage with and without trophoblastic component. Also, there has been no study investigating the relevance of trophoblastic differentiation NMIBC for risk of recurrence and progression.

Another manifestation of divergent differentiation is neuroendocrine variant which is most frequently represented by small cell carcinoma. Small cell carcinoma accounts for approximately $1 \%$ of all bladder tumours and is rarely seen in non-muscle invasive stage (62). This variant can coexist not only with conventional UC, but also with squamous or glandular component. Most patients present with advanced or metastatic disease at the time of diagnosis (63). Thus, any amount of small cell component has to be reported as there is a need for early aggressive treatment, also for non-muscle invasive disease. There is an evidence that neoadjuvant chemotherapy followed by RC represents the optimal treatment option (64).

\section{Molecular variants in non-muscle invasive bladder cancer}

Over the recent years, many attempts have been made to identify molecular markers that would help not only to improve risk assessment for tumour recurrence or progression, but also to predict response to treatment and eventually to establish new, targeted treatment strategies. The constant improvement in immunochemical and genomic techniques resulted in identification of multiple markers, such as expression of different cell cycle genes, receptor tyrosine kinases, angiogenetic factors, cytokeratins, cell adhesion genes, mRNA and microRNA expression signatures (65-67). Several studies evaluated the clinical utility of these biomarkers in NMIBC, with P53, P27, Rb, Ki-67, FGFR3 being the most thoroughly investigated ones (67). Some of them were found to have prognostic value, but their ability to predict oncological outcomes with sufficient precision was limited (68-70). Thus, it has been postulated that a panel of biomarkers could improve their predictive accuracy, yet, the further studies assessing this matter reported conflicting results (71-73).

Nevertheless, the research in the field of molecular markers have been useful in determining specific gene expression profiles that have helped to define subgroups within bladder cancer. Similar approach was used in breast cancer, where molecular subdivision to four distinct groups was found to have significant prognostic impact (74). Over the last years, different research teams independently identified a variety of subtypes of bladder cancer based on RNA and immunohistochemical expression characteristics $(75,76)$. Most of this work has been carried out in MIBC, where relationship between expression subgroups and outcome as well as treatment response has been found (75-77). The data regarding molecular subclassification in NMIBC is less extensive $(78,79)$.

One of the earliest attempts to classify bladder cancer in terms of molecular characteristics was made by Lindgren et al., who analyzed 144 samples, including both muscle invasive and non-muscle invasive lesions, and identified two molecular subtypes of MS1 and MS2 (75). It was shown that MS1 tumours presented with frequent FGFR3/PIK3CA mutations and were mainly Ta, while MS2 cancers were $\geq \mathrm{T} 2$ and characterized by TP53/MDM2 alterations and RB1 losses, as well as higher rates of genomic instability. Both Ta and T1 tumours were further divided into two distinct subtypes. By using two different algorithms, researchers were able to define specific gene signatures that were predictive for progression and survival (75).

This concept was further extended by the Lund University team which included a group of $200 \mathrm{Ta}$ and T1 tumours out of total 308 cancers (78). After wide gene expression profiling and immunohistochemical evaluation, five subtypes of bladder cancer were defined-urobasal A and B, genomically unstable, squamous-like and infiltrated. The urobasal A group was characterized by KRT5 and FGFR3 overexpression, while urobasal B group shared 
features of urobasal A and the other subtypes. Genomically unstable tumors were harboring TP53 mutations and ERBB2 overexpression, whereas a squamous cell carcinoma-like subtype was recognized by squamous cell differentiation, overexpression of basal keratins. Eventually, a group of infiltrated tumors was distinguished, characterized by infiltration of immunologic cells and extracellular matrix gene expression. Importantly, these molecular subtypes showed distinct survival patterns in which urobasal A showed good prognosis, genomically unstable and the infiltrated group intermediate, and the urobasal B and the squamous-like carried the worst prognosis. There was no strict overlap between defined molecular subtypes and pathologic stage. Even though most of Ta, G1 and G2 tumors were predominantly of the urobasal A subtype, all of molecular subtypes were found among T1 tumors and no fundamental differences were observed between invasive and non-muscle invasive genomically unstable cases (78).

In a subsequent study, an extensive immunohistochemical mapping of subtype-defining markers was performed (80). It was shown that the subtypes can be characterized by distinct features at the immunohistochemical level. This led to development of immunohistochemical/histologybased classifier that corresponds well with mRNA classification and can be easily implemented in a standard pathologic evaluation $(80,81)$. Subsequently, Patschan et al. performed analysis of 167 stage T1 tumours in order to assess if molecular subtyping can provide with improved prognostic information. All tumours were characterized by immunohistochemical classifier and categorized into the three molecular subtypes: urobasal, genomically unstable and squamous-cell-carcinoma-like. The analysis revealed significantly higher frequencies of multifocal tumors, concomitant CIS, lymphovascular invasion, and deeper invasion depths in genomically unstable and squamouslike subtypes compared to urobasal tumors. Genomically unstable and squamous-like tumors were also associated with high risk of progression (82).

Later on, Hedegaard et al. $(77,79)$ distinguished three major classes within NMIBC characterized by basal- and luminal-like features, previously described in the setting of MIBC. A comprehensive transcriptional analysis of total 460 patients with mainly $\mathrm{Ta}$ and $\mathrm{T} 1$ tumours was performed and revealed that class 1 and class 2 tumours show luminal-like characteristics, but demonstrate different levels of aggressiveness, and class 3 tumours show basallike characteristics (79). Class 1 and 2 lesions showed high expression of uroplakins, which are mainly expressed in luminal cells, whereas KRT5 and KRT15 expression, markers of undifferentiated basal cells, were primarily found in class 3 cancers. Furthermore, mutations in wellknown cancer driver genes, such as TP53 and ERBB2, were primarily found in high-risk tumours, together with APOBEC-related mutational signatures, mainly in class 2 tumours (79). The expression of APOBEC proteins has been previously associated with poor prognosis in bladder cancer (83). Interestingly, a significant concordance between three classes and the Lund taxonomy was found. Class 1 tumors were primarily classified as urobasal A (71\%), class 3 tumors were also classified as urobasal A $(65 \%)$, and class 2 tumors were classified as infiltrated $(37 \%)$ or genomically unstable (57\%) (71). Tumors of high stage and grade, concomitant CIS, and progression to MIBC were more frequently observed in classes 2 and 3 than in class 1 . Similarly, patients with EORTC high clinical risk were predominantly found in classes 2 and 3. It was concluded that classes 2 and 3 represent high-risk tumors, whereas class 1 tumors have good prognosis (79).

Subsequently, Hurst et al. defined different molecular subtypes within non-muscle invasive bladder carcinoma (84). A group of 140 stage Ta tumors was analyzed and two genomic subtypes were identified. Genomic subtype 1 (GS1) which was characterized by containing no or few copy-number alterations and genomic subtype 2 (GS2) that showed high frequency of chromosome 9 deletions. Additionally, GS2 tumors presented with upregulated glycolysis, DNA repair and mTORC1 signaling, which may serve as potential therapeutic targets. The analysis revealed a significant difference in overall survival and some evidence of decreased recurrence-free survival in patients with GS2 tumors, however, the difference was not statistically significant. All tumors expressed GATA3, FOXA1 and PPARG genes which are features of luminal status. There has also been a strong overlap between analyzed tumors and the Urobasal A subgroup of Lund classification (83). Additionally, There was compatibility of GS2 tumors with the class 2 cancers as defined by Hedegaard et al. $(79,84)$.

In a study by van Kessel et al., a cohort of 1,239 patients with NMIBS was prospectively evaluated (85). Tumor samples were analyzed for GATA2, TBX2, TBX3, and ZIC4 methylation and FGFR3, TERT, PIK3CA, and RAS mutation status. Patients were also divided into three groups of low, intermediate and high risk of progression based on EORTC nomogram. Progression was seen in 57 patients with only one case in low-risk group and 45 in high-risk tumors. It was also found that GATA2 and TBX3 
hypermethylation and FGFR3 wild-type mutation were significantly associated with higher risk of progression. The EORTC high-risk group was further classified into good, moderate and poor classes, according to FGFR3 mutations and the GATA2 methylation status. Only $2.1 \%$ of patients in high-risk good class progressed to muscle-invasive stage, while in patients with high-risk poor subtype progression occurred in $14.9 \%$. It was concluded that the combination GATA2 methylation and FGFR3 mutation status with EORTC risk system improves risk stratification in noninvasive bladder carcinoma and allows classification of highrisk tumors into three subtypes with a low, medium and high risk of progression and different clinical prognoses (85).

Although there is a diversity of published classifications and different teams used different definitions for describing molecular subtypes, there is a significant overlap among them. Efforts have been made in order to standardize the terminology for these subtypes. To date, a consensus among experts exists to use the term Basal/Squamous-like in describing tumors that display the expression of basal keratins and an enrichment of tumors with histological squamous differentiation. It was also suggested that the term Urothelial-like should replace previously used Urobasal subtype (86). Recently, a consensus classification of molecular subtypes in muscle-invasive bladder cancer has been proposed. Based on available studies, authors identified six molecular subtypes; luminal papillary, luminal non-specified, luminal unstable, stroma-rich, basal/ squamous and neuroendocrine-like. This may facilitate further investigations regarding clinical usefulness of such classification in prospective manner (87). Currently there is no such consensus in terms of molecular classification of NMIBC.

\section{Molecular changes in some variant histology urothelial carcinomas}

Although different variants of bladder cancer were found to share some molecular similarities, certain histological patterns of urothelial carcinoma can be characterized by specific genetic aberrations.

The link between plasmocytoid differentiation and loss of E-cadherin expression has been found, which is related to mutation in the $\mathrm{CDH} 1$ gene as reported by $\mathrm{Al}$-Ahmadie et al. $(88,89)$. It was found that truncating mutations in the CDH1 gene occur in $84 \%$ of plasmacytoid carcinomas and are pathognomonic to this histologic variant (83).

Strong association between micropapillary urothelial carcinoma and ERBB2 gene amplification and HER2 overexpression has been reported $(90,91)$. The ERBB2 amplification in micropapillary urothelial carcinoma occurs more frequently than in classic urothelial carcinoma and several reports associated it with worse cancer-specific survival following radical cystectomy (92). This may have important clinical implications as therapeutic agents targeting HER2 signaling are broadly used in the treatment of breast cancer (93). The downregulation of miR-296 and activation of chromatin-remodeling complex RUVBL1 have also been found to characterize micropapillary differentiation, but the role of these alterations is not clearly understood and requires further investigations (94).

In nested variant of bladder cancer, TERT promoter mutations were found to be useful in distinguishing between nested pattern and benign urothelial lesions (95). This mutational status was linked to poor prognosis in sarcomatoid variant of urothelial carcinoma (96). High rates of TERT promoter mutations were also found in neuroendocrine bladder carcinoma (97). This histological variant was also found to be associated with frequent cooccurrence of TP53 and RB1 mutations as well as with the absence of KDM6A truncating mutations, CDKN2A deletion and CCND1 amplifications, which are commonly seen in urothelial carcinoma (97).

Micropapillary, nested, plasmacytoid variants were found to have luminal features, whereas urothelial carcinoma with squamous or neuroendocrine differentiation was found to have basal characteristics (98). In general, basal tumors tend to be enriched with squamous and small cell/neuroendocrine features and inactivating mutations as well as deletions of TP53 and RB1, while luminal tumors are often enriched with papillary histopathological features and activating mutations in FGFR3, ERBB3 mutations and ERBB2 amplifications (99). Overexpression of FOXA1 together with overexpression of GATA3 and PPARG activation were also found to be markers of luminal status (100). Warrick et al. proposed that tumors can be distinguished into luminal and basal subtypes based on FOXA1 and CK14 expression (98). The rates of CK15 expression were higher in squamous variant of bladder cancer compared to micropapillary, nested, and plasmacytoid carcinomas, marking its basal status. Inversely, micropapillary, nested, and plasmacytoid cancers expressed higher levels of FOXA1 and low rates of CK15, suggesting their luminal character (98). As shown by Hedegaard et al., subtyping of non-muscle invasive bladder cancer based on luminal and basal characteristics can have significant 
prognostic utility (79). Further studies are required to evaluate if such distinction is of clinical usefulness.

\section{Discussion}

The need for improving the accuracy of predictive and prognostic tools in NMIBC is undisputable. Current prognostic models rely only on clinical and pathologic data and do not reflect the biological landscape of the disease.

The accurate identification of variant histology implicates patient prognosis and inform right treatment decisions. It has to be noted that the number of patients with variant histology is in fact not that small. Kamat et al. suggested that almost $45-50 \%$ of variant histology cases are missed during pathologic evaluation after TURB, and variant pattern that was not reported after initial electroresection was found in cystectomy specimens in about half of the cases (47). In a study by Shah et al. (40), TURB specimens were reviewed once again before the initiation of therapy. It was shown that variant histology was not reported in $44 \%$ of cases.

Most studies on histological variants of bladder cancer suggest a more aggressive clinical course than conventional UC. Overall risk of recurrence and progression is higher than in conventional UC, even when detected in nonmuscle invasive stage. However, when treated aggressively, survival outcomes are comparable to those achieved in pure UC $(34,45,58,61)$. The controversy regarding optimal management is fuelled by the fact that most of recommendations are based on retrospective series, with small case number reported or extrapolation of data from pure UC. In variant histology NMIBC management with endoscopic resection followed by intravesical treatment has shown inferior performance when compared to conventional UC. Only glandular differentiation has shown promising response to intravesical BCG (25). To date, radical cystectomy is considered the mainstay of treatment in variant histology bladder carcinoma (101). The application of chemotherapy is controversial, although tumors with neuroendocrine components, like small cell carcinoma, and plasmocytoid tumors, appear to be chemosensitive and the use of neoadjuvant chemotherapy should be taken into consideration $(58,64)$.

Emerging genomic informations are expected to complement clinical and pathological data and change the paradigms in the management of bladder cancer. There is no doubt that different molecular classification systems improved our understanding of bladder cancer biology and confirmed its heterogeneous nature. Several reports highlighted the clinical significance of molecular stratification of bladder cancer, but the available evidence is based on retrospective data $(78,79,82,85)$. Molecular subtyping gives promise not only for improving risk assessment, but also in predicting response to BCG or chemotherapy. Finally, molecular alterations might become targets for novel drugs to improve the overall response of these patients (82). However, several obstacles have been linked to molecular subtyping. The traditional system that uses clinical and pathologic parameters provides quick estimation of prognosis and is convenient to operate in clinical practice. Future developments should focus on adjusting molecular classifications to the requirements of daily practice. Secondly, during the monitoring and followup of non-muscle invasive disease, recurrent tumors can present with different molecular features than the primary tumor (11). The implication of that is that every tumor detected during follow-up should undergo thorough pathological and molecular evaluation to better stratify the risk of progression. This can generate additional cost to the whole diagnosis and treatment process. Eventually, different sites in each tumor can present features of different molecular subtypes which can significantly affect the accuracy of molecular stratification (12). Thus, molecular information should be interpreted with caution, together with clinical and pathologic data, before any decisions regarding treatment are undertaken.

Nevertheless, progress has been made and molecular subtyping will surely gain more recognition as a promising tool in risk stratification. However, its implementation into clinical practice requires further validation in prospective trials, especially in the context of non-muscle invasive bladder cancer.

\section{Acknowledgments}

Funding: None.

\section{Footnote}

Reporting Checklist: The authors have completed the Narrative Review reporting checklist. Available at http:// dx.doi.org/10.21037/tcr-20-2257

Conflicts of Interest: All authors have completed the ICMJE uniform disclosure form (available at http://dx.doi. org/10.21037/tcr-20-2257). The authors have no conflicts 
of interest to declare.

Ethical Statement: The authors are accountable for all aspects of the work in ensuring that questions related to the accuracy or integrity of any part of the work are appropriately investigated and resolved.

Open Access Statement: This is an Open Access article distributed in accordance with the Creative Commons Attribution-NonCommercial-NoDerivs 4.0 International License (CC BY-NC-ND 4.0), which permits the noncommercial replication and distribution of the article with the strict proviso that no changes or edits are made and the original work is properly cited (including links to both the formal publication through the relevant DOI and the license). See: https://creativecommons.org/licenses/by-nc-nd/4.0/.

\section{References}

1. Bertz S, Hartmann A, Knüchel-Clarke R, et al. Specific types of bladder cancer. Pathologe 2016;37:40-51.

2. Humphrey PA, Moch H, Cubilla AL, et al. The 2016 WHO Classification of Tumours of the Urinary System and Male Genital Organs-Part B: Prostate and Bladder Tumours. Eur Urol 2016;70:106-19.

3. Burger M, Catto JW, Dalbagni G, et al. Epidemiology and risk factors of urothelial bladder cancer. Eur Urol 2013;63:234-41.

4. Colt JS, Friesen MC, Stewart PA, et al. A case-control study of occupational exposure to metalworking fluids and bladder cancer risk among men. Occup Environ Med 2014;71:667-74.

5. Gouda I, Mokhtar N, Bilal D, et al. Bilharziasis and bladder cancer: a time trend analysis of 9843 patients. J Egypt Natl Canc Inst 2007;19:158-62.

6. Figueroa JD, Ye Y, Siddiq A, et al. Genome-wide association study identifies multiple loci associated with bladder cancer risk. Hum Mol Genet 2014;23:1387-98.

7. Kramer MW, Altieri V, Hurle R, et al. Current Evidence of Transurethral En-bloc Re-section of Nonmuscle Invasive Bladder Cancer. Eur Urol Focus 2017;3:567-76.

8. Sylvester RJ, Brausi MA, Kirkels WJ, et al. Longterm efficacy results of EORTC genitourinary group randomized phase 3 study 30911 comparing intravesical instillations of epirubicin, bacillus Calmette-Guérin, and bacillus Calmette-Guérin plus isoniazid in patients with intermediate- and high-risk stage Ta $\mathrm{T} 1$ urothelial carcinoma of the bladder. Eur Urol 2010;57:766-73.
9. Sylvester RJ, Van der Meijden APM, Oosterlinck W, et al. Predicting recurrence and progression in individual patients with stage Ta $\mathrm{T} 1$ bladder cancer using EORTC risk tables: a combined analysis of 2596 patients from seven EORTC trials. Eur Urol 2006;49:466.

10. Fernandez-Gomez J, Madero R, Solsona E, et al. Predicting nonmuscle invasive bladder cancer recurrence and progression in patients treated with bacillus Calmette-Guerin: the CUETO scoring model. J Urol 2009;182:2195.

11. Meeks JJ, McConkey DJ. Limited Upstaging in Luminal Subtype Tumors: Ready for Clinical Practice? Eur Urol 2019;76:207-8.

12. Zhu S, Yu W, Yang X, et al. Traditional Classification and Novel Subtyping Systems for Bladder Cancer. Front Oncol 2020;10:102.

13. Warrick JI, Sjödahl G, Kaag M, et al. Intratumoral Heterogeneity of Bladder Cancer by Molecular Subtypes and Histologic Variants. Eur Urol 2019;75:18-22.

14. Wasco MJ, Daignault S, Zhang Y, et al. Urothelial carcinoma with divergent histologic differentiation (mixed histologic features) predicts the presence of locally advanced bladder cancer when detected at transurethral resection. Urology 2007;70:69-74.

15. Cai T, Tiscione D, Verze P, et al. Concordance and clinical significance of uncommon variants of bladder urothelial carcinoma in transurethral resection and radical cystectomy specimens. Urology 2014;84:1141-6.

16. Moschini M, Shariat SF, Freschi M, et al. Is transurethral resection alone enough for the diagnosis of histological variants? A single-center study. Urol Oncol 2017;35:528.e1-5.

17. Abufaraj M, Shariat SF, Foerster B, et al. Accuracy and prognostic value of variant histology and lymphovascular invasion at transurethral resection of bladder. World $\mathrm{J}$ Urol 2018;36:231-40.

18. Mostafa MH, Sheweita SA, O'Connor PJ, et al. Relationship between schistosomiasis and bladder cancer. Clin Microbiol Rev 1999; 12:97-111.

19. Dahm P, Gschwend JE. Malignant non-urothelial neoplasms of the urinary bladder: a review. Eur Urol 2003;44:672-81.

20. Locke JR, Hill DE, Walzer Y, et al. Incidence of squamous cell carcinoma in patients with long-term catheter drainage. J Urol 1985;133:1034-5.

21. Stein JP, Skinner EC, Boyd SD, et al. Squamous cell carcinoma of the bladder associated with cyclophosphamide therapy for Wegener's granulomatosis: 
a report of 2 cases. J Urol 1993;149:588-9.

22. Lagwinski N, Thomas A, Stephenson AJ, et al. Squamous cell carcinoma of the bladder: a clinicopathologic analysis of 45 cases. Am J Surg Pathol 2007;31:1777-87.

23. Lopez-Beltran A, Requena MJ, Alvarez-Kindelan J, et al. Squamous differentiation in primary urothelial carcinoma of the urinary tract as seen by MAC387 immunohistochemistry. J Clin Pathol 2007;60:332-5.

24. Li G, Hu J, Niu Y, et al. Squamous differentiation in pT1 bladder urothelial carcinoma predicts poor response for intravesical chemotherapy. Oncotarget 2017;9:217-23.

25. Gofrit ON, Yutkin V, Shapiro A, et al. The Response of Variant Histology Bladder Cancer to Intravesical Immunotherapy Compared to Conventional Cancer. Front Oncol 2016;6:43.

26. Antunes AA, Nesrallah LJ, Dall'Oglio MF, et al. The role of squamous differentiation in patients with transitional cell carcinoma of the bladder treated with radical cystectomy. Urol 2007;33:339-45; discussion 346.

27. Honma I, Masumori N, Sato E, et al. Local recurrence after radical cystectomy for invasive bladder cancer: an analysis of predictive factors. Urology 2004;64:744-8.

28. Izard JP, Siemens DR, Mackillop WJ, et al. Outcomes of squamous histology in bladder cancer: a population-based study. Urol Oncol 2015;33:425.e7-13.

29. Scosyrev E, Yao J, Messing E, et al. Urothelial carcinoma versus squamous cell carcinoma of bladder: is survival different with stage adjustment? Urology 2009;73:822-7.

30. Solomon JP, Lowenthal BM, Kader AK, et al. Challenges in the diagnosis of urothelial carcinoma variants: can emerging molecular data complement pathology review? Urology 2017;102:7-16.

31. Grignon DJ, Ro JY, Ayala AG, et al. Primary adenocarcinoma of the urinary bladder. A clinicopathologic analysis of 72 cases. Cancer 1991;67:2165-72.

32. López-Beltrán A, Martin J, Garcia J, et al. Squamous and glandular differentiation in urothelial bladder carcinomas. Histopathology, histochemistry and immunohistochemical expression of carcinoembryonic antigen. Histol Histopathol 1988;3:63-8.

33. Chalasani V, Chin JL, Izawa JI, et al. Histologic variants of urothelial bladder cancer and nonurothelial histology in bladder cancer. Can Urol Assoc J 2009;3:S193-8.

34. Miller JS, Epstein JI. Noninvasive urothelial carcinoma of the bladder with glandular differentiation: report of 24 cases. Am J Surg Pathol 2009;33:1241-8.

35. Shapur NK, Katz R, Pode D, et al. Is radical cystectomy mandatory in every patient with variant histology of bladder cancer. Rare Tumors 2011;3:e22.

36. Mitra AP, Bartsch CC, Bartsch G Jr, et al. Does presence of squamous and glandular differentiation in urothelial carcinoma of the bladder at cystectomy portend poor prognosis? An intensive case-control analysis. Urol Oncol 2014;32:117-27.

37. Kim SP, Frank I, Cheville JC, et al. The impact of squamous and glandular differentiation on survival after radical cystectomy for urothelial carcinoma. J Urol 2012;188:405-9.

38. Yorozuya $W$, Nishiyama N, Shindo T, et al. Bacillus Calmette-Guérin may have clinical benefit for glandular or squamous differentiation in non-muscle invasive bladder cancer patients: ret-rospective multicenter study. Jpn J Clin Oncol 2018;48:661-6.

39. Torenbeek R, Blomjous CE, de Bruin PC, et al. Sarcomatoid carcinoma of the urinary bladder. Clinicopathologic analysis of 18 cases with immunohistochemical and electron microscopic findings. Am J Surg Pathol 1994;18:241-9.

40. Shah RB, Montgomery JS, Montie JE, et al. Variant (divergent) histologic differentiation in urothelial carcinoma is under-recognized in community practice: impact of mandatory central pathology review at a large referral hospital. Urol Oncol 2013;31:1650-5.

41. Lopez-Beltran A, Pacelli A, Rothenberg HJ, et al. Carcinosarcoma and sarcomatoid carcinoma of the bladder: clinicopathological study of 41 cases. J Urol 1998;159:1497-503.

42. Wright JL, Black PC, Brown GA, et al. Differences in survival among patients with sarcomatoid carcinoma, carcinosarcoma and urothelial carcinoma of the bladder. J Urol 2007;178:2302-6; discussion 2307.

43. Black PC, Brown GA, Dinney CP, et al. The impact of variant histology on the outcome of bladder cancer treated with curative intent. Urol Oncol 2009;27:3-7.

44. Black PC, Brown GA, Dinney CP, et al. Clinical and therapeutic significance of aberrant differentiation patterns in bladder cancer. Expert Rev Anticancer Ther 2007;7:1015-26.

45. Vetterlein MW, Wankowicz SAM, Seisen T, et al. Neoadjuvant chemotherapy prior to radical cystectomy for muscle-invasive bladder cancer with variant histology. Cancer 2017;123:4346-55.

46. Wang JK, Boorjian SA, Cheville JC, et al. Outcomes following radical cystectomy for micropapillary bladder cancer versus pure urothelial carcinoma: a matched cohort analysis. World J Urol 2012;30:801-6. 
47. Kamat AM, Dinney CP, Gee JR, et al. Micropapillary bladder cancer: a review of the University of Texas M. D. Anderson Cancer Center experience with 100 consecutive patients. Cancer 2007;110:62-7.

48. Willis DL, Fernandez MI, Dickstein RJ, et al. Clinical outcomes of cT1 micropapillary bladder cancer. J Urol 2015;193:1129-34.

49. Spaliviero M, Dalbagni G, Bochner BH, et al. Clinical outcome of patients with T1 micro-papillary urothelial carcinoma of the bladder. J Urol 2014;192:702-7.

50. Jackson BL, Mohammed A, Mayer N, et al. Is Immediate Radical Cystectomy Necessary for All Patients with NonMuscle-Invasive Micropapillary Bladder Cancer? Urol Int 2016;96:32-8.

51. Abufaraj M, Foerster B, Schernhammer E, et al. Micropapillary Urothelial Carcinoma of the Bladder: A Systematic Review and Meta-analysis of Disease Characteristics and Treatment Outcomes. Eur Urol 2019;75:649-58.

52. Witjes, JA, Babjuk M, Bellmunt J, et al. EAU-ESMO Consensus Statements on the Management of Advanced and Variant Bladder Cancer-An International Collaborative Multistakeholder. Eur Urol 2020;77:223-50.

53. Borhan WM, Cimino-Mathews AM, Montgomery EA, et al. Immunohistochemical differentiation of plasmacytoid urothelial carcinoma from secondary carcinoma involvement of the bladder. Am J Surg Pathol 2017;41:1570-5.

54. Keck B, Stoehr R, Wach S, et al. The plasmacytoid carcinoma of the bladder - rare variant of aggressive urothelial carcinoma. Int J Cancer 2011;129:346-54.

55. Li Q, Assel M, Benfante NE, et al. The Impact of Plasmacytoid Variant Histology on the Survival of Patients with Urothelial Carcinoma of Bladder after Radical Cystectomy. Eur Urol Focus 2019;5:104-8.

56. Dayyani F, Czerniak BA, Sircar K, et al. Plasmacytoid urothelial carcinoma, a chemosensitive cancer with poor prognosis, and peritoneal carcinomatosis. J Urol 2013;189:1656-61.

57. Holmäng S, Johansson SL. The nested variant of transitional cell carcinoma--a rare neoplasm with poor prognosis. Scand J Urol Nephrol 2001;35:102-5.

58. Wasco MJ, Daignault S, Bradley D, et al. Nested variant of urothelial carcinoma: a clinicopathologic and immunohistochemical study of 30 pure and mixed cases. Hum Pathol 2010;41:163-71.

59. Mally AD, Tin AL, Lee JK, et al. Clinical outcomes of patients with $\mathrm{T} 1$ nested variant of urothelial carcinoma compared to pure urothelial carcinoma of the bladder. Clin Genitourin Cancer 2017. DOI: 10.1016/ j.clgc.2017.07.002.

60. Douglas J, Sharp A, Chau C, et al. Serum total HCG beta level is an independent prognostic factor in transitional cell carcinoma of the urothelial tract. Br J Cancer 2014;110:1759-66.

61. Martin JE, Jenkins BJ, Zuk RJ, et al. Human chorionic gonadotrophin expression and histological findings as predictors of response to radiotherapy in carcinoma of the bladder. Virchows Arch A Pathol Anat Histopathol 1989;414:273-7.

62. Wang G, Xiao L, Zhang M, et al. Small cell carcinoma of the urinary bladder: a clinicopathologic and immunohistochemical analysis of 81 cases. Hum Pathol 2018;79:57-65.

63. Celik O, Ekin G, Ipekci T, et al. Diagnosis and treatment in primary bladder small cell carcinoma: Literature review. Arch Ital Urol Androl 2016;88:52-5.

64. Lynch SP, Shen Y, Kamat A, et al. Neoadjuvant chemotherapy in small cell urothelial cancer improves pathologic downstaging and long-term outcomes: results from a retrospective study at the MD Anderson Cancer Centre. Eur Urol 2013;64:307-13.

65. Shariat SF, Weizer AZ, Green A, et al. Prognostic value of P53 nuclear accumulation and histopathologic features in T1 transitional cell carcinoma of the urinary bladder. Urology 2000;56:735-40.

66. Shariat SF, Ashfaq R, Sagalowsky AI, et al. Predictive value of cell cycle biomarkers in non-muscle invasive bladder transitional cell carcinoma. J Urol 2007;177:481-7; discussion 487.

67. Soria F, Krabbe LM, Todenhöfer T, et al. Molecular markers in bladder cancer. World J Urol 2019;37:31-40.

68. Zhou X, Zhang G, Tian Y, et al. p53 Status correlates with the risk of recurrence in non-muscle invasive bladder cancers treated with Bacillus Calmette-Guérin: a metaanalysis. PLoS One 2015;10:e0119476.

69. van Rhijn BW, Zuiverloon TCM, Vis AN, et al. Molecular Grade (FGFR3/MIB-1) and EORTC risk-scores are predictive in primary non-muscle invasive bladder cancer. Eur Urol 2010;58:433-41.

70. Shariat SF, Bolenz C, Godoy G, et al. Predictive value of combined immunohistochemical markers in patients with pT1 urothelial carcinoma at radical cystectomy. J Urol 2009;182:78-84.

71. Hernández S, Lopez-Knowles E, Lloreta J, et al. FGFR3 and Tp53 mutations in T1G3 transitional 
bladder carcinomas: independent distribution and lack of association with prognosis. Clin Cancer Res 2005;11:5444-50.

72. Passoni N, Gayed B, Kapur P, et al. Cell-cycle markers do not improve discrimination of EORTC and CUETO risk models in predicting recurrence and progression of nonmuscle-invasive high-grade bladder cancer. Urol Oncol 2016;34:485.e7-14.

73. Alkhateeb SS, Neill M, Bar-Moshe S, et al. Long-term prognostic value of the combination of EORTC risk group calculator and molecular markers in non-muscle-invasive bladder cancer patients treated with intravesical Bacille Calmette-Guérin. Urol Ann 2011;3:119-26.

74. Sørlie T, Perou CM, Tibshirani R, et al. Gene expression patterns of breast carcinomas distinguish tumor subclasses with clinical implications. Proc Natl Acad Sci U S A 2001;98:10869-74.

75. Lindgren D, Frigyesi A, Gudjonsson S, et al. Combined gene expression and genomic profiling define two intrinsic molecular subtypes of urothelial carcinoma and gene signatures for molecular grading and outcome. Cancer Res 2010;70:3463-72.

76. Choi W, Porten S, Kim S, et al. Identification of distinct basal and luminal subtypes of mus-cle-invasive bladder cancer with different sensitivities to frontline chemotherapy. Cancer Cell 2014;25:152-65.

77. Robertson AG, Kim J, Al-Ahmadie H, et al. Comprehensive Molecular Characterization of MuscleInvasive Bladder Cancer. Cell 2017;171:540-556.e25.

78. Sjödahl G, Lauss M, Lövgren K, et al. A molecular taxonomy for urothelial carcinoma. Clin Cancer Res 2012;18:3377-86.

79. Hedegaard J, Lamy P, Nordentoft I, et al. Comprehensive transcriptional analysis of early-stage urothelial carcinoma. Cancer Cell 2016;30:27-42.

80. Lindgren D, Sjödahl G, Lauss M, et al. Integrated genomic and gene expression profiling identifies two major genomic circuits in urothelial carcinoma. PLoS One 2012;7:e38863.

81. Sjödahl G, Lövgren K, Lauss M, et al. Toward a molecular pathologic classification of urothelial carcinoma. Am J Pathol 2013;183:681-91.

82. Patschan O, Sjödahl G, Chebil G, et al. A Molecular Pathologic Framework for Risk Stratification of Stage T1 Urothelial Carcinoma. Eur Urol 2015;68:824-32; discussion 835-6.

83. Nordentoft I, Lamy P, Birkenkamp-Demtroder K, et al. Mutational context and diverse clonal development in early and late bladder cancer. Cell Rep 2014;7:1649-63.

84. Hurst CD, Alder O, Platt FM, et al. Genomic subtypes of non-invasive bladder cancer with distinct metabolic profile and female gender bias in KDM6A mutation frequency. Cancer Cell 2017;32:701-715.e7.

85. van Kessel KE, van der Keur KA, Dyrskjot L, et al. Molecular Markers Increase Precision of the European Association of Urology Non-Muscle-Invasive Bladder Cancer Progression Risk Groups. Clin Cancer Res 2018;24:1586-93.

86. Lerner SP, McConkey DJ, Hoadley KA, et al. Bladder Cancer Molecular Taxonomy: Summary from a Consensus Meeting. Bladder Cancer 2016;2:37-47.

87. Kamoun A, de Reyniès A, Allory Y, et al. A Consensus Molecular Classification of Muscle-invasive Bladder Cancer. Eur Urol 2020;77:420-33.

88. Al-Ahmadie HA, Iyer G, Lee BH, et al. Frequent somatic CDH1 loss-of-function mutations in plasmacytoid variant bladder cancer. Nat Genet 2016;48:356-8.

89. Lim MG, Adsay NV, Grignon DJ, et al. E-cadherin expression in plasmacytoid, signet ring cell and micropapillary variants of urothelial carcinoma: comparison with usual-type high-grade urothelial carcinoma. Mod Pathol 2011;24:241-7.

90. Tschui J, Vassella E, Bandi N, et al. Morphological and molecular characteristics of HER2 amplified urothelial bladder cancer. Virchows Arch 2015;466:703-10.

91. Fleischmann A, Rotzer D, Seiler R, et al. Her2 amplification is significantly more frequent in lymph node metastases from urothelial bladder cancer than in the primary tumours. Eur Urol 2011;60:350-7.

92. Schneider SA, Sukov WR, Frank I, et al. Outcome of patients with micropapillary urothelial carcinoma following radical cystectomy: ERBB2 (HER2) amplification identifies patients with poor outcome. Mod Pathol 2014;27:758-64.

93. Bang YJ, Van Cutsem E, Feyereislova A, et al. Trastuzumab in combination with chemotherapy versus chemotherapy alone for treatment of HER2-positive advanced gastric or gastro-oesophageal junction cancer (ToGA): a phase 3, open-label, randomised controlled trial. Lancet 2010;376:687-97.

94. Guo CC, Dadhania V, Zhang L, et al.Gene Expression Profile of the Clinically Aggressive Micropapillary Variant of Bladder Cancer. Eur Urol 2016;70:611-20.

95. Zhong M, Tian W, Zhuge J, et al. Distinguishing nested variants of urothelial carcinoma from benign mimickers by TERT promoter mutation. Am J Surg Pathol 
2015;39:127-31.

96. Wang X, Lopez-Beltran A, Osunkoya AO, et al. TERT promoter mutation status in sarcomatoid urothelial carcinomas of the upper urinary tract. Future Oncol 2017;13;705-14.

97. Shen P, Jing Y, Zhang R, et al. Comprehensive genomic profiling of neuroendocrine bladder cancer pinpoints molecular origin and potential therapeutics. Oncogene 2018;37:3039-44.

98. Warrick JI, Kaag M, Raman JD, et al. FOXA1 and CK14 as markers of luminal and basal subtypes in histologic variants of bladder cancer and their associated conventional urothelial carcinoma. Virchows Arch 2017;471:337-45.

99. Choi W, Czerniak B, Ochoa A, et al. Intrinsic basal and luminal subtypes of muscle-invasive bladder cancer. Nat Rev Urol 2014;11:400-10.

100. Warrick JI, Walter V, Yamashita H, et al. FOXA1, GATA3 and PPARy Cooperate to Drive Luminal Subtype in Bladder Cancer: A Molecular Analysis of Established Human Cell Lines. Sci Rep 2016;6:38531.

101.Lobo N, Shariat SF, Guo CC, et al. What Is the Significance of Variant Histology in Urothelial Carcinoma? Eur Urol Focus 2020;6:653-63.

Cite this article as: Piszczek R, Krajewski W, Moschini M, Kołodziej A, Nowak Ł, Poterek A, Zdrojowy R. Combination of histological and molecular data for improving outcome prediction in non-muscle invasive bladder cancer-narrative review. Transl Cancer Res 2020;9(11):7323-7336. doi: 10.21037/ tcr-20-2257 